\title{
O Cerro Aconcágua (6959m, Argentina): um destino de turismo de Aventura: um alto lugar mítico e simbólico
}

\author{
The Cerro Aconcagua (6959m, Argentina: an Adventure Touristic \\ Destination: a mythical and symbolical high place
}

\section{Le Cerro Aconcagua (6959 m, Argentine): destination du tourisme d'aventure, haut lieu mythique et symbolique}

Michel Raspaud ${ }^{1}$

\begin{abstract}
Resumo
Este artigo busca compreender o modo de organização e de funcionamento de um destino do turismo de aventura de alta altitude. Após relembrar o conteúdo do conceito de destino turístico, descreve o exemplo histórico de Chamonix. Em seguida, através do método da observação participante, no âmbito de uma expedição amadora, ele análisa a organização concreta in situ do destino de Aconcágua. Em conclusão, para além dos serviços propostos, este artigo sublinha que, para a clientela de turistas alpinistas, o preço de um tal destino reporta-se, ao senso simbólico atribuído a ele, as provas físicas e mentais suportadas, e a sociabilidade própria ao meio cultural do mundo do alpinismo.
\end{abstract}

Palavras-chave: destino turístico; turismo de aventura; Aconcágua; valores simbólicos; sociabilidade.

\begin{abstract}
The aim of this article is to understand the organization and working process of a high altitude touristic destination. After the definition of the concept of touristic destination, it describes the historic example of Chamonix. Then, by the way of the observing participation method, realized during an amateur expedition, the concrete organization in situ of the Aconcagua destination is analysed. In conclusion, the author underlines the fact that, more than the services offered, the price of such a destination is, for the mountaineer clients, in the symbolic sense given to the destination, in the physical and mental suffering tried out, and the lived sociability of the cultural mountaineering world.
\end{abstract}

Keywords : touristic destination; adventure tourism; Aconcagua; symbolic values; sociability.

\section{Résumé}

Cet article vise à comprendre le mode d'organisation et de fonctionnement d'une destination du tourisme d'aventure de haute altitude. Après avoir rappelé le contenu du concept de destination touristique, il décrit l'exemple historique de Chamonix. Puis, par la méthode de l'observation participante, dans le cadre d'une expédition amateur, il analyse l'organisation concrète in situ de la destination Aconcagua. En conclusion, au-delà des services proposés, il souligne que le prix d'une telle destination renvoie, pour la clientèle des touristes alpinistes,

\footnotetext{
${ }^{1}$ Doutor em Sociologia do Esporte. Professor Ttular da Université Joseph Fourier, Grenoble, France. E-mail : michel.raspaud@ujf-grenoble.fr
} 
au sens symbolique qui lui est attribuée, aux épreuves physiques et mentales endurées, et à la sociabilité vécue propre au milieu culturel de monde de l'alpinisme.

Mots clés: Destination touristique - Tourisme d'aventure - Aconcagua - Valeurs symboliques - Sociabilité.

\section{Introduction}

Depuis une trentaine d'années maintenant, ce qui était auparavant réservé à une élite sportive, professionnnelle et/ou sociale, le tourisme sportif ou tourisme d'aventure, s'est largement diffusé dans les populations relativement privilégiées des pays les plus riches de la planète (Amérique du nord, Europe occidentale, Japon), mais aussi dans ceux qui accèdent petit à petit à cette catégorie, le Brésil par exemple, pour une partie de leurs populations tout au moins.

Ici, les facteurs favorables ne seront pas analysés, car largement connus : augmentation du pouvoir d'achat, amélioration générale des conditions de vie (habitat, santé) et de travail (développement du secteur tertiaire, réduction du temps de travail, allongement de la durée des congés), accès à l'éducation et aux loisirs, inscription du sport et de l'éducation physique dans les cursus scolaires et politiques volontaristes des Etats, développement de la pratique sportive comme facteur de santé et inscription dans la vie quotidienne des populations, médiatisation du spectacle et des vedettes (stars) du sport...

Ainsi, en très peu de temps, sous forme d'abord associative puis rapidement commerciale, dans les années 1970 et 1980, et surtout dans les années 1990, le trekking (dans les Alpes, l'Himalaya, puis dans le reste du monde : Andes, Patagonie, Afrique...) est-il devenu, en France tout du moins, une niche économique représentant toutefois plusieurs dizaines de millions d'euros de chiffre d'affaire pour des sociétés organisatrices de voyages sportifs telles que « Terre d'Aventures », «Atalante », «Allibert », etc. Concomitament, des expéditions commerciales ont trouvé un public (c’est-à-dire une clientèle), certes relativement restreint, mais concret, passionné, souvent compétent contrairement à ce que l'on en entend dire, et surtout solvable pour aller en Himalaya, dans les Andes, ou ailleurs...

Ainsi, des montagnes comme le Kilimandjaro, le Chimborazo, l'Aconcagua, le Cho Oyu et l'Everest constituent-elles des destinations prisées du tourisme sportif ou du tourisme d'aventure, et peuvent être dorénavant envisagées comme des « classiques », même si elles ne 
sont ni banales et ni sans risque, et demandent à la fois compétence et prudence, l'altitude imposant ses exigences à la physiologie.

L’intervention se découpra en quatre temps :

- Avant tout, il est nécessaire de définir le concept de destination touristique, tel que l'entendent les spécialistes. Ce concept de destination touristique sera appliqué à deux cas, éloignés dans le temps et dans l'espace, mais exemplaires : le Mont-Blanc et l'Aconcagua.

- Ainsi, il sera montré dans quelles conditions, Chamonix, petit village montagnard retiré de Savoie avant que ce Duché appartenant au Royaume de Piémont-Sardaigne ne devienne français, est devenu "La Mecque” de l'alpinisme et, par ce fait, une destination du tourisme d'aventure.

- Puis, l’Aconcagua sera envisagé, même sans habitat permanent (contrairement à Chamonix), comme une destination du tourisme d'aventure.

- Enfin, d’un point de vue sociologique, il sera mis en évidence que les infrastructures matérielles et les réseaux d'acteurs pour important qu'ils soient ne suffisent pas, et qu'il faut que le social, le relationnel, le symbolique (ce que Baudrillard appelait “valeur d'échange”, en opposition à la “valeur d'usage”) (BAUDRILLARD, 1970), circulent, aient toute leur place, soient efficients pour qu'une destination touristique fonctionne.

\section{Qu'est-ce Qu'une Destination Touristique?}

Du point de vue strictement marketing, la destination touristique est un concept qui se décline en fonction des marchés. Ainsi, un lieu touristique prendra ou perdra la dimension de destination selon les segments de marché considérés, dans des zones de chalandise données (GIBSON, 1999). Une destination touristique est donc un lieu de forte concentration touristique vis-à-vis duquel les touristes attendent des avantages qui constitueront les déterminants de sa capacité d'attraction touristique. Une destination peut être alors, en fonction de son extension spatiale, un site, une station, une région, voire un pays tout entier (WEBER, 1998). Autrement dit, toujours en demeurant dans le domaine pragmatique, concret, une destination constitue la simple projection d'une économie touristique forte, et structurée (HOERNER, 1997). Ceci semble corroborer l'assertion selon laquelle « tout lieu est potentiellement touristique ». A la limite, il suffirait d’y créer un service qui corresponde à la demande (CHADEFAUD, 1988). 
Cependant, si pour le touriste, la destination touristique est également un espace image, porteur de valeurs, elle est également un espace de consommation multiproduits avec une gestion cohérente. Ainsi, le touriste, de la même manière que le client dans un supermarché, navigue à travers un univers de prestations dans lequel il réalise lui-même son panier, son cocktail (CORNU, 1996). Il existe donc de multiples scénarii de consommation, d’autant plus que les facteurs influençant le comportement et la prise de décision de la clientèle touristique sont nombreux : les influences du marketing mix, les influences psychologiques, socioculturelles, de situation, etc. (DUBOIS e JOLIBERT, 1998).

Sur le lieu de la destination touristique, le consommateur va se trouver face aux options suivantes :

- Certains achats ont un caractère obligatoire pour ne pas dire obligé, comme se loger, se nourrir. On parlera donc de produit obligé.

- D’autres sont de véritables déclencheurs de fréquentation de la destination touristique, de son attractivité. Il s’agit des produits souhaités ou désirés. Le plus souvent, ce sont les éléments qui déterminent le choix final du consommateur.

- Enfin, certains achats ne sont pas prévus lorsque le consommateur arrive sur la destination touristique. Ces produits, qui au départ sont inconnus, vont donner lieu à des achats d’impulsion, de découverte.

En résumé, la destination touristique est un lieu de rencontre entre une offre (dont l'espace n'est qu'une composante, au même titre qu'une prestation) et une clientèle (touristes et résidents), qu'il faut savoir comprendre et connaître afin de se situer au plus près de ses attentes. Cette attention de tous les instants reste particulièrement délicate en raison du nécessaire positionnement du produit tant dans le domaine de l'affectif que du rationnel. Mais une destination touristique est également un système - ou ordre local (FRIEDBERG , 1993) - où l'ensemble des acteurs s’inscrivent dans un partenariat obligé, en vue de la réussite d'un projet global et commun. Un projet de destination touristique doit donc s’attacher à servir le «bien commun». Par conséquent, il ne sera pas spécifiquement privé ou public :

- Dans le contexte d'une concurrence internationale, il n’y a plus de destination unique à laquelle aucune autre ne pourrait se substituer. 
- La compétition entre destinations s’appuie en premier lieu sur les prix pratiqués ; elle est également liée à d’autres facteurs tels que la sécurité, la paix civile, les bonnes conditions sanitaires (la «bonne gouvernance» exercée par les pouvoirs publics).

- L’image valorisante du pays, que doivent construire les pouvoirs publics et sur laquelle les opérateurs peuvent s'appuyer pour commercialiser leurs produits (RAHARINOSY e RASPAUD , 2005).

\section{Chamonix et la Construction Historique d'une Destionation du Tourisme d'Aventure}

En 1741, le village de Chamonix n'est qu'une bourgade de montagne de la Savoie, difficilement accessible par un itinéraire risqué du fait du mauvais état de la voirie, des à pics vertigineux, mais aussi du brigandage qui sévit. Il est totalement coupé du monde l’hiver. Jusqu'à ce que, cette année-là, il reçoive la visite de deux voyageurs Anglais, Wyndham et Pocock qui s’extasient devant le spectacle féerique que constituent les formes figées extraordinaires du glacier appelé « Mer de glace ». Comme cela se pratique à l’époque dans l'aristocratie et chez les élites sociales, ils écrivent et envoient de nombreuses lettres à l'ensemble de leurs relations, et le fait devient un objet de discussions mondaines.

Chamonix en subira un premier effet au cours des années qui suivent : un afflux, tout à fait relatif quand même, de voyageurs qui viendront visiter et admirer cette merveille, nécessitant et conduisant alors à une amélioration des conditions d'accueil d'une part, invitant quelques personnes sinon à se spécialiser, du moins à être capables d'accompagner les visiteurs sur les lieux d'autre part.

A cette époque, il faut noter que sous l'impulsion des idées rousseauistes, la représentation de la nature se transforme: elle devient lieu de beauté et d'admiration, mais aussi de régénérescence physique et morale. La ville, espace sécurisé par ses remparts devenant, progressivement et inversement, le lieu du miasme et de la promiscuité, sans compter déjà le congestionnement du trafic urbain comme le décrit Louis-Sébastien Mercier (1788).

Moins d’un demi-siècle plus tard, un second événement, plus considérable encore, construit définitivement la vocation de Chamonix. Sous l'impulsion d'Horace Benedict de Saussure (par l'offre d'une somme d'argent à qui y parviendrait ou trouverait um itinéraire d'accès), savant genevois qui voulait réaliser des relevés scientifiques à son sommet, deux chamoniards, le cristalier et chasseur de chamois Jacques Balmat et le médecin MichelGabriel Paccard, parviennent à la cime du Mont Blanc, en fin d'après-midi, le 8 août 1786. 
Là aussi, un certain nombre de représentations de la montagne auront dû être dépassées pour que cette ascension se réalise : espace utilitaire, la montagne est, dans l'imaginaire populaire des autochtones, peuplée d'être terrifiants (dragons, monstres...), et l'on ne ressort pas vivant d’une nuit passée en haute montagne... Ce que Jacques Balmat fit plus ou moins malgré lui...

La nouvelle de l'ascention est alors répandue rapidement dans toutes les elites européennes, par le même mode de diffusion que précédemment, et renforce ainsi la position de Chamonix.

Ce n’est, toutefois, qu’après la Révolution de 1789, et surtout la fin de l'épisode napoléonien dont les guerres ensanglantent tout le continent européen que Chamonix se construira comme destination touristique par ses splendeurs naturelles et son objectif de sport-aventure ${ }^{2}$ :

- Augmentation de la capacite d’accueil,

- Amélioration des conditions d’hébergement,

- Spécialisation et hiérarchisation de "professionnels” de la montagne (guides, porteurs),

- Sous l’impulsion de la municipalité, organisation et structuration de la “profession”, dans la première Compagnie de guides dès 1823 (avec instauration du "tour de rôle” pour garantir le travail à tous et la création d'une caisse de solidarité),

- Constitution de réseaux d’acteurs ayant des interêts communs (hôteliers, commerçants, guides, porteurs...),

Mise en place de rituels et de symboles :

- Départ matinal des ascensionnistes et de la caravane devant l'ensemble des villageois rassemblés,

- Accompagnement par quelques villageois de l'expédition jusqu’à une certaine limite au-delà de laquelle ne s’aventure que la caravane,

- Suivi de la progression de l'expédition à la longue vue depuis le village ou depuis les montagnes alentours,

- Coups de canons tirés au village pour annoncer l’arrivée de la caravane au sommet du Mont Blanc,

\footnotetext{
${ }^{2}$ Le terme "sport" est ici employé par commodité, celui-ci étant en train de se constituer à la même époque en Angleterre.
} 
- Retour et défilé dans le village sous les vivas de la foule (la réussite d’une expédition est à chaque fois un exploit et un événement qui conforte la notoriété et renforce le pouvoir d'attractivité de Chamonix),

- Les ascensionnistes offrent traditionnellement un repas de gala, dans l'auberge ou l'hôtel qui les accueillent, aux guides et porteurs,

- Le syndic du village remet aux ascensionnistes un document officiel attestant de leur réussite sous certification des guides ${ }^{3}$.

\section{Le Cerro Aconcagua (6962 m.) une Destionation Contemporaine du Tourisme d'Aventure ${ }^{4}$}

Bien que n'ayant pas d'habitat / habitant permanent sur l'ensemble de l'année, le Cerro Aconcagua doit cependant être considéré comme une destination du tourisme d'aventure, au sens du concept de la science du tourisme.

En premier lieu, il s'agit d'une attraction, par le fait que c'est la plus haute montagne des Amériques et du monde en dehors de l'Asie ${ }^{5}$, et que sa face Sud d'une élévation de près de 3000 mètres est l'une des plus gigantesques, impressionnantes... et prestigieuses du monde pour les alpinistes. Le pied de la voie normale, comme de la face Sud sont d'un accès relativement aisé, en deux jours de marche depuis Horcones par Confluencia (camp intermédiaire, à $3300 \mathrm{~m}$ d'altitude) : environ $36 \mathrm{~km}$ pour Plaza de Mulas (4300 m) et $25 \mathrm{~km}$ pour Plaza Francia (4100 m). Une autre entrée, par Punta de Vacas, est moins fréquentée (60 km de marche), et conduit à Plaza Argentina (4200 m), autre point de départ pour l'ascension. Tout concourt à faire de Plaza de Mulas (bien plus que Plaza Argentina) une destination:

- Des structures d'accueil : un refuge-hôtel et un camp de base, ouverts pendant la saison (15 novembre / 31 mars) ;

- Les conditions d'hébergement : le refuge-hôtel d'une capacite de 80 places, avec cuisine, et douches chaudes (payantes) ; au camp de base, des tentes collectives installées à demeure durant la saison par chaque compagnie de tourisme-aventure ;

\footnotetext{
${ }^{3}$ L'ensemble de ces éléments est issu de l'excellent ouvrage de l'historien Philippe JOUTARD, 1986.

${ }^{4}$ Les informations qui suivent sont issues d'une observation participante, dans le cadre d'une expédition amateure auto-organisée, du 12 janvier au 10 février 2007.

${ }^{5}$ On a d'ailleurs pendant longtemps estimé l'altitude de l'Aconcagua supérieure à 7000 mètres, limite symbolique significative.
} 
- Une offre de restauration (alimentation, boisson) au refuge comme au camp de base ;

- Des structures de communication : téléphone de la compagnie nationale argentine, internet, courier postal ;

- La vente de souvenirs : cartes postales, livres, posters, tee-shirts, jusqu’à la présence d'um peintre qui vend ses propres posters et lithographies des lieux ;

- Un service de transport de bagages, par mûles et mûletiers interposés, depuis Horcones jusqu’à Confluencia, puis Plaza de Mulas (refuge ou camp de base) ou Plaza Francia ; il sert aussi de service de ravitaillement pour le refuge et le camp de base ;

- Un service de porteurs, à pied, pour les différents camps d’altitude utilisables (Nido de Condores à 5350 m, Berlin à 5850 m).

Au-delà de ces services minimum que les acteurs de l'offre proposent aux clients/touristesaventuriers, une structure de coordination, en terme de contrôle, de surveillance et de sécurité, a été mise em place par le Parque et les Autorités provinciales à laquelle participent et coopèrent l'ensemble des acteurs commerciaux :

- Un controle des entrées et sorties du Parque par les employés de celui-ci, plus pour des raisons de sécurité (une personne qui rentre et qui ne ressort pas est considérée potentiellement comme en situation de danger... ou morte !), et des raisons financières (en 2006-2007, le permis d'ascension coûtait US\$ 300) ${ }^{6}$;

- Un service public de police pour relever et constater officiellement les accidents (et les morts), et déclencher les secours ;

- La distribution et récupération de sacs en plastique pour les déchets afin de maintenir en l'état le capital nature, et donc l'attractivité touristique des lieux (à l'opposé, on peut considérer la route entre Mendoza et Punta del Inca - environ 180 km - comme, probablement, la plus longue déchèterie à ciel ouvert du monde !) ;

- Un service privé de médecins en contrat avec le Parque, à Confluencia comme au camp de base où il possèdent un local spécialement aménagé. Ces médecins se déplacent au refugehôtel, et à Nido de Condores si nécessaire, et sont en relation avec l'Hôpital de Mendoza (service spécialisé) pour la précision d'un diagnostic, ou bien l'évacuation d'un malade ou d'un blessé. Leur activité principale consiste toutefois en l'information et la mise en garde des touristes sur les conditions de l'ascension (l’Aconcagua, malgré son altitude, est présenté

\footnotetext{
${ }^{6}$ US\$120 en 1999-2000, US\$ 160 en 2000-2001 (GASQUES, 2002, p. 84).
} 
comme aussi exigeant qu'un sommet de 8000 m en Himalaya ${ }^{7}$, eu égard aux conditions atmosphériques qui y règnent), mais surtout à surveiller la santé et l'état physique des alpinistes (fréquence cardiaque, tension artérielle, oxygénation du sang, état pulmonaire, gelures éventuelles...) et, si nécessaire, retarder une tentative d'ascension étant donné le mauvais acclimatement, ou l'interdire (en faisant alors signer une décharge de responsabilité par le condidat au sommet), ou encore en faisant rapatrier la personne sur l'Hôpital de Mendoza ;

- Un service privé d’hélicoptères en contrat avec le Parque pour ravitailler la Police et les gardiens du Parque en station à Plaza de Mulas et à Nido de Condores, intervenant pour aller chercher malades et blessés à Plaza de Mulas et à Nido de Condores, et les descendre au camp de base ou à l'entrée du Parque où ils se voient pris en charge par leur structure privée (on peut supposer que dans un cas très grave, la personne peut être immédiatement et directement conduite à l’Hôpital de Mendoza).

\section{Le Cerro Aconcagua: du Mythe, du Symbolique, du Social}

Cependant, jusqu’à présent, l’Aconcagua a été présenté uniquement à travers les services touristiques qui sont offerts à la clientèle des alpinistes. En tant que tel, il constitue donc un lieu touristique, un espace de consommation au sein duquel la clientèle peut, va, doit dépenser de l'argent, et créer une économie. Mais, il faut bien tenir compte du fait que ce ne sont pas les services qui attirent les alpinistes (même si, reconnaissons-le, ils incitent grandement à leur venue et facilitent leur vie quotidienne), mais bien quelque chose qui préexiste aux services touristiques, à savoir : la montagne.

En effet, s’il n’y avait pas de services touristiques (hébergement, transport, télécommunication, sécurité...), les touristes d'aventure, c'est-à-dire les candidats à l'ascension de l'Aconcagua, viendraient quand même. Certes, bien moins nombreux (4285 entrées en 1999-2000, 4850 en 2000-2001 ${ }^{8}, 6490$ en 2004-2005 ${ }^{9}$ ) dans des conditions bien moins confortables, mais ils seraient là, au camp de base, pour tenter l'ascension de la plus haute montagne des Amériques, un mythe.

\footnotetext{
${ }^{7}$ Cette façon de présenter l'Aconcagua est ambigue: est-ce du nationalisme pur et simple, une stratégie marketing pour valoriser la destination, ou une stratégie médicale pour engager les alpinistes à la prudence et à la raison?

${ }^{8}$ GASQUES, 2002, p. 81- 257.

${ }^{9}$ PARQUE PROVINCIAL ACONCAGUA, S/D.
} 
Quelques faits obervés, et que les photographies présentées dans le diaporama illustrent, montrent à l'évidence que le mythe, le symbolique, le social en définitive, sont les éléments organisant l'intérêt pour l'Aconcagua, et le faisant accéder au statut de destination touristique.

\subsection{L’Aconcagua, un espace hors du monde, un espace sacré}

Il existe deux portes d'entrée pour l'Aconcagua, et deux seulement : Horcones, et Punta de Vacas. Au-delà du contrôle exercé par les employés du Parque, de la distribution des sacs en plastique, de l'attribution d'un numéro, il s'agit aussi d'une barrière symbolique qui fait pénétrer le trekkeur ou l'alpiniste dans un endroit réservé, séparé, au sein duquel n’importe qui ne s'engage pas sans s'y être un minimum préparé, d'une manière ou d'une autre (organisation du voyage et de la logistique ; lecture des livres contant les ascensions anciennes ou récentes, les hauts faits et exploits, les drames aussi ; mise en condition psychologique ; achat et préparation de son propre matériel...). Pour signifier cette coupure, Peter Habeler, qui fut le premier homme avec Reinhold Messner à atteindre le sommet de l'Everest sans utilisation d’oxygène artificiel, le 8 mai 1978, déclarait à propos de la cascade de glace qui se trouve au-delà du camp de base, et qui donne accès à la Combe Ouest (Western Cwm), voie d'ascension népalaise, que celle-ci marque la différence entre le trekkeur et l'alpiniste, sépare les enfants des grandes personnes... (HABELER, 1979).

Cette réserve, cette séparation matérialisée par l'entrée dans le Parque, se concrétise également, sur le terrain, par l'épreuve que constitue la marche d'approche. La plus ou moins grande difficulté de celle-ci, sa longueur (36 km à pied), la nécessité impérieuse de gérer l'acclimatement de son corps à l'altitude (on ne passe pas de 2800 m à 4200 m en 24 heures sans conséquences physiologiques), imposent au trekkeur et à l'alpiniste um rythme lent qui contribue à renforcer le sentiment de sacralité du lieu, des montagnes, des paysages qu'il a tout le temps d'admirer. On ne se précipite pas au devant de l'Aconcagua, la condition humaine doit se plier aux exigences de fait qu'impose la montagne au corps de l'alpiniste.

Aussi, la montée à Plaza de Mulas se réalise en trois jours minimum, pour une première étape dans le processus d'acclimatement.

Après une première journée de marche qui conduit au camp intermédiaire de Confluencia (3300 m), le second jour l’occasion est recommandée aux alpinistes d'aller visiter la face Sud de l'Aconcagua, l'une des parois les plus impressionnantes du monde (près de $3000 \mathrm{~m}$ de hauteur). 
Celle-ci a été gravie pour la première fois en 1954 par une expédition française, et l’on se rend sur le lieu du camp de base de l'époque (Plaza Francia, 4100 m, matérialisée par un panneau) pour admirer la paroi et, d'une certaine manière, se prosterner mentalement devant le lieu de multiples exploits au cours de ce dernier demi siècle. En effet, les plus grands alpinistes sont venus ici ouvrir leur propre voie d'ascension qui, bien sûr, porte leur nom, comme la voie Messner par exemple, du nom du premier alpiniste à avoir gravi les quatorze sommets de plus de 8000 m en Himalaya, et sans oxygène.

Lorsque tout se passe bien, le troisième jour, une longue et harassante journée de marche (8 à 10 h, 24 km, 1000 m de dénivelée, compte non tenu des conditions météorologiques) conduit les alpinistes de Confluencia à Plaza de Mulas (4300 m), au Camp de base ou au Refugehôtel, au pied même de la voie normale d'ascension. Là aussi, la dimension de la montagne, la hauteur de la paroi, d'autant plus impressionnante que l'alpiniste est à ses pieds, invite celuici à concevoir l'Aconcagua comme autre chose qu'un tas de pierres, un amoncellement de rochers inertes recouverts de neige et de glace. Au contraire, cela contribue à l'apprécier comme une entité, un être ayant sa vie propre, sa volonté, ses soubressauts, ses crises, une forme de pouvoir surnaturel auquel l'alpiniste devra s'affronter, se confronter, s'il veut parvenir à ses fins, atteindre le sommet...

A cet égard, les alpinistes, pourtant des êtres qui doivent faire preuve de rationalité, bien souvent guides de haute montagne pour une bonne partie d'entre eux, qui ont donc été instruits des dangers objectifs de la montagne dans le cadre de leur formation (chutes de pierres, avalanches, risques induits par les conditions métérologiques, etc.), dans leurs récits (et au-delà d'une volonté narrative consciente de construire l'action comme l'affrontement à un être surnaturel), essentialisent la montagne, lui accorde une pensée, une volonté, et généralement de mal faire. Elle est présentée comme un monstre ${ }^{10}$ (eu égard à ses dimensions, ses difficultés d'ascension, les risques et dangers qu'elle cache), qui en veut aux hommes (et aux femmes) qui viennent en quelque sorte la profaner. S'il y a profanation, c'est bien qu'il y a du sacré (ELIADE, 1965).

\subsection{L’Aconcagua, un Mythe}

Le mythe, selon Claude Lévi-Strauss, est une histoire que la société se raconte à elle-même, et qui dit quelque chose d’elle-même (LÉVI-STRAUSS, 1962). Il en est ainsi pour l’Aconcagua,

\footnotetext{
${ }^{10}$ On peut retenir quelques titres pour illustrer ce propos. En ce qui concerne l'Aconcagua (GASQUES, 2002 e AYMONE, 2008). Mais, surtout, NICLEVICZ, 2007. Sur cet aspect particulier, Roland Barthes a bien montré comment les journalistes sportifs essentialisent les difficulties rencontrées par les coureurs dans les courses cyclistes, en constituant ces difficultés comme des êtres maléfiques, des monstres, etc. (BARTHES, 1958).
} 
en particulier à travers la littérature du genre qui participe à la constitution de ce mythe : nombreux sont les ouvrages qui en rapportent les ascensions et les exploits, les drames, les figures héroïques, les figures malheureuses aussi, les actions de solidarité et de secours. L’Aconcagua se constitue ainsi en un concentré d'histoires humaines, petites ou grandes, plus ou moins glorieuses, et qui s’impriment dans la mémoire des alpinistes, participant ainsi à la construction de leurs représentations du monde, et en particulier des montagnes, de l’alpinisme, des alpinistes, de leurs valeurs, et... de l’Aconcagua!

Quelques faits qui organisent les intétrêts des alpinistes pour tenter l'ascension de l’Aconcagua :

(i) En premier lieu, la question de l'altitude: longtemps, l'Aconcagua a été crédité d'une altitude supérieure à 7000 m (7035 m, puis 7010 m), tout comme en Afrique le Kilimandjaro était identifié comme supérieur à 6000 m (6010m ; 5895 m en réalité). Il y a, ici, par ces chiffres ronds, des limites symboliques qui sont instituées : le trekkeur, l'alpiniste dépassera une frontière d'altitude, atteindra par ses propres moyens un niveau d'élévation relativement au niveau de la mer, qui signifie quelque chose, comme une étape dans une éventuelle progression vers des zones plus élevées par exemple.

(ii) En second lieu, la question de l'histoire, toujours très présente chez les alpinistes, d'autant plus présente que les sommets sont élevés et prestigieux. Cela participe de l'inscription de l'alpiniste dans une série, commencée avant lui par les exploits des pionniers pour réaliser la « première » (c'est à dire parvenir le premier au sommet et y attacher son nom pour l'éternité), dans lesquels il met symboliquement ses pas (et, dans notre monde, rares sont les occasions de se sentir en communion par delà le temps avec les pionniers), et qui se poursuivra après lui...

- La permière ascension (en solitaire) par le guide Suisse Matthias Zurbgiggen, le 14 janvier 1897, dans le cadre de l'expédition du britannique Edward Fitz Gerald.

- La première ascension de la face Sud par une expédition française en 1954.

- L'ouverture (en solitaire pour la partie sommitale) d'une voie nouvelle dans cette même paroi par Reinhold Messner, le 23 janvier 1974 (seulement 14 voies ont été ouvertes dans la face Sud en 46 ans, entre 1954 et 2000).

- Qui plus est, entre 1926 et 2000, 104 personnes sont mortes dans leur tentative, réussie ou non, de l'ascension de l'Aconcagua (GASQUES, 2002, p. 74-100). L'annonce d'une telle statistique rend d'autant plus valeureuse la tentative, et plus encore la réussite. 
Ainsi, en janvier 1994, Lucien Berardini, leader avec Guido Magnone de l'expédition française à la face Sud en 1954, est revenu pour fêter le quarantenaire de l'ascension, avec Hugues Beauzille. Mais, estimant les conditions de neige trop dangereuse, Berardini est allé sur la voie normale, pendant que Beauzille demeurait au Sud, joignant ses forces à celles de Pierre Griscelli (guide Corse) et sa cliente Céline Rambaud. Toutefois, pris dans la tempête, ils ne purent redescendre, sauf Griscelli qui parvint à donner l'alerte après deux jours d'errance dans la face. Lorsque lorsque les secours pervinrent à la tente, Hugues Beauzille était mort d'épuisement, Céline Rambaud atteinte de gelures (ECHEVARRIA, 1996, p. 224).

Plus important, encore, fut le drame de février 1998, lorsque trois alpinistes Brésiliens, Mozart Catão (35 ans) et Alexandre Oliveira (24 ans), tous deux de Teresópolis, et Othon Leonardos (23 ans), moururent dans un avalanche dans la face Sud. Mozart Catão était considéré come l’um dês deux ou trois meilleurs alpinistes Brésiliens, pour son adresse, sa vigueur et son expérience. Il avait un motif particulièrement important de tenter la face Sud de l’Aconcágua, qui était de dépasser son déclaré, le Paranense Waldemar Niclevicz. Les deux alpinistes avaient gravi le même jour l'Everest (le 14 mai 1995), mais, de retour au Brésil, Mozart Catão accusa son partenaire d'omettre son nom l'entreprise. A partir de ce jour, ils ne se parlèrent plus. Pour Catão, Oliveira et Leonardos, l'ascension de la face Sud signifiait qu’ils seraient les premiers Brésiliens à réussir cet exploit.

Les faits, tels qu’ils ont été rapportés dans la presse :

- Le $1^{\text {er }}$ jour, 31 janvier : Alexandre Oliveira, Mozart Catão et Othon Leonardos n’atteignent pás le lieu prévu pour passer la nuit (camp envisagé à 5200m) et passent la nuit à 4800m.

- Les $2^{\mathrm{e}}$ et $3^{\mathrm{e}}$ jours ( ${ }^{\mathrm{er}}$ et 2 février), ils atteignent le glacier supérieur, et une crevasse à 5800 où ils passent la nuit. La tempête de neige se poursuit. L’équipe brésilienne attend un jour de plus sur le lieu.

- Le $4^{\mathrm{e}}$ jour (3 février), les alpinistes reprennent leur ascension, malgré le mauvais temps. Aprés avoir grimpé 600 mètres, ils décident de bivouaquer. Une avalanche les emporte à 8 heures du soir.

Lorsque l'accident a lieu, ils se trouvent à 6150m d'altitude, à peine à 800m de leur objectif. Mozart et Alexandre sont emportés au bas de la montagne. Othon reste pendu par une corde de 60 mètres, dans le vide, le long de la paroi gelée. Dans cette position, il parvient à sortir la radio de son sac, à composer le numéro de fréquence du Parc provincial de l’Aconcagua, avec lequel ses compagnon du camp de base sont en relation. Suspendu dans l'abîme, avec les 
jambes brisées par l'impact de la corde au moment de l'avalanche, il converse avec Dálio et Ronaldo par radio pendant 2h30, avant de mourir de froid.

\subsection{L’Aconcagua, un Haut Lieu Symbolique}

Haut lieu topographique, l'Aconcagua est donc aussi haut lieu symbolique. Les alpinistes viennent personnellement chercher quelque chose sur l'Aconcagua : la confirmation de leurs capacités d’alpiniste, un défi physique et mental, une performance, une confirmation de leurs qualités psychologiques, acquérir ou confirmer une reputation, franchir une étape dans leur carrière d'alpiniste, etc.

Relativement à cette symbolique de l'Aconcagua, le Refuge-hôtel constitue, à lui seul, en tant que construction en dur, un lieu de permanence, qui perdure au-delà du temps et à qui l'on peut confier ses réussites, ses défaites, ses hommages aux personnes aimées, chères, ou disparues sur la montagne ou en montagne (ami, frère, compagnon, etc.). Ainsi, le refuge constitue une sorte de mausolée, un panthéon de souvenirs, où l'on vient accrocher, suspendre les signes du souvenir de son propre exploit, de sa propre souffrance, du souvenir aussi de celui / celle qui n’est plus...

Ainsi, le refuge est parsemé, dans les différents lieux qui constituent son espace public, de multiples signes d'appartenance nationale, locale, sportive. Cependant, le réfectoire, la salle ou l'on déjeune, dîne, mais aussi où l'on se rencontre pour boire un thé, lire, jouer aux cartes, discuter, constitue à cet égard un lieu unique, à la forte charge émotionnelle.

Cette salle est au sens premier du terme un sanctuaire (c'est-à-dire un lieu saint, sanctifié) dans lequel on vient placarder sur les murs, suspendre au plafond, tout un ensemble d'objets significatifs de l’identité de chacun et/ou du collectif, annonçant la réussite, informant de la performance (on a seulement atteint Berlin [5850 m]...), de la difficulté et de la souffrance subies dans une ascension inachevée (oedème...), et du souvenir de celui, celle, ceux qui ne sont pas revenus. C’est la salle des reliques...

Cette salle, le réfectoire, plus que tout autre lieu sur l'Aconcagua, même le sommet, est celui qui inscrit littéralement l'alpiniste dans l'histoire au quotidien, celle de l'anonyme, venu ici pour s'éprouver, et qui laisse la trace de cette épreuve, plus ou moins joyeuse, à demeure, pour les générations suivantes. 


\subsection{L’Aconcagua, un Lieu de Sociabilité}

Mais la montagne est avant tout un lieu de sociabilité et d'échange entre les alpinistes : discuter, raconter des histoires d'alpinisme et celles concernant cette montagne en particulier (transmission de vive voix de la culture de l'alpinisme, et construction d'um sentiment d'appartenance et d'identité propre à ce monde-là ; intégration, petit à petit, du langage, du jargon de l'alpinisme ; acceptation de ses normes et mise aux normes.

Mais aussi, la fonction importante, voire primordiale de la diffusion d'informations sur l'état de la montagne, ses difficulties techniques d'ascension, la qualité du rocher, les conditions de la neige et de la glace, les conditions météorologiques, comme le souligne à juste titre Viviane Seigneur (2007).

C'est aussi, en particulier sur des sommets aussi internationaux que l'Aconcagua, la rencontre avec d'autres nationalités, le découverte de manières d'être différentes et, dans le même temps, de manière incoonsciente, la recherche ou la confirmation des stéréotypes nationaux.

Ainsi, par exemple, à l'intérieur du Refuge-hôtel, uniquement en observant les fanions, teeshirts, auto-collants et autres marques du passage des différentes expéditions qui y ont été laissés comme trace du passage ou reliques, 42 nationalités différentes différentes ont pu être identifiées en janvier-février 2007.

Il faut s’arrêter, ici, sur un fait significatif : la sociabilité produite par le gérant du refuge de Plaza des Mulas et son équipe (second, filles et garçons de salle, cuisinière, ces dernières par la qualité des repas fournis... et la satisfaction de l'estomac, en haute montagne, dans des conditions de température et parfois de farniente obligatoire, est un élément important du moral). La bonne humeur, la pratique aisée de plusieurs langues (espagnol, français - presque parfait dû à plusieurs saisons de travail dans un refuge des Pyrénées -, anglais...) permettant l'échange avec tout le monde, la fête organisée pour chaque ascension de l'Aconcagua (champagne argentin offert par le gérant), c ontribue à produire une ambiance dse sociabilité qui déteint sur les pensionnaires.

\subsection{Valeur d'usage et Valeur d'échange (Baudrillard).}

La valeur d'usage de l'Aconcagua, c'est la tentative d'ascension à près de 7000m d'altitude. Vaincre l'altitude (adaptation physiologique à l'air raréfié), dépasser la fatigue due à l'effort physique (l'épuisement consécutif à de longues heures de marche : 10, 12, 15 heures selon les cas, les étapes, le jour du sommet étant le plus épuisant et dangereux, à cause de l'euphorie de 
la montée vers le sommet d'abord, à cause de la fatigue lors de la descente ensuite), réaliser une performance sportive (préparation physique et mentale).

La valeur d'échange constituée par l'Aconcagua, par exemple, c'est l'entrée dans le monde de la très haute altitude ; c'est le voyage vers une destination lointaine ; ce sont les récits que l'on en fait après le retour chez soi à travers les photographies qui sont montrées et longuement commentées ; ce peut être, aussi, une sorte d'envie que l'on crée chez les autres... et qui va faire se poursuivre le cycle de l'alpinisme...

\section{Conclusion}

Les observations réalisées dans le cadre de cette expédition à l’Aconcagua, en janvier-février 2007, mettent en évidence plusieurs aspects :

D’abord, bien que les alpinistes se considèrent généralement avant tout comme des sportifs, ils doivent être appréhendés essentiellement comme des touristes. Touristes-aventuriers, certes, si l'on veut reprendre la formule des sciences du tourisme et du sport, mais touristes quand même ${ }^{11}$, c'est-à-dire une clientèle qui consomme, dépense de l'argent, et produit une économie. Les prestataires privés du tourisme sportif qui interviennent dans le périmètre de l’Aconcagua l'ont bien compris, et les autorités publiques également, qui ont mis en place des structures et une organisation qui, dans le cadre d'une protection de l'environnement d'une part, mais aussi du capital-image que constitue l'Aconcagua d'autre part, conserve en l'état la capacité d'attraction de la destination (équipe médicale, équipe de secours).

Ensuite, une destination touristique de haute altitude comme l'Aconcagua, étant donné les conditions particulières dans lesquelles on y séjourne (vie sous tente au camp de base, ou dans le refuge-hôtel, et dans le camps d'altitude avec les montées et descenters inévitables), et la durée de ce séjour (quinze jours à trois semaines), produit de la sociabilité qui demeure un élément important dans les souvenirs des aventuriers touristes.

Enfin, et c'est peut-être l'élément le plus important de la destination, chacun vient y chercher (et y apporte : cf. les reliques et étendards de toutes sortes apposés dans le refectoire et l'ensemble de refuge, constitué en véritable sanctuaire), chacun vient y chercher et y apporte donc du symbolique à travers l'épreuve - au sens initiatique du terme (RASPAUD, 2003) -

\footnotetext{
${ }^{11}$ En français, le terme « touriste » est péjoratif et, généralement, l’on n’aime pas se faire traiter de « touriste ». Qui plus est, le sportif qui se rend à l'Aconcagua, sommet de près de $7000 \mathrm{~m}$ d'altitude, s'engage en terme d'alpinisme vers une destination " classante » (pas autant que l'Everest, bien sûr) qui procure une « distinction » au sens où l'entend Pierre Bourdieu, et qui ne souffre donc pas la confusion (BOURDIEU, 1979).
} 
que constitue l'ascension et la montée en très haute altitude. Quelqu'en soit le résultat, rares sont ceux qui ne laissent pas une partie d'eux-mêmes ${ }^{12}$, vêtement sur lequel on inscrit son nom, qui identifie son origine, éléments auxquels on rajoute une date (celle de la réussite ou de l'échec), autocollant, drapeau national ou régional, fanion du club, etc. Et l'on sait que le symbolique n’a pas de prix, et c'est bien ce qui fait son prix...

\section{Bibliographie}

AYMONE Assis. Aconcágua. 2008. O cume e depois morrer, o ser e a montanha. Rio de Janeiro / São Paulo, Editora Record.

BARTHES Roland. 1958. Mythologies. Paris, Seuil.

BAUDRILLARD Jean. 1970. La société de consommation, ses mythes, ses structures. Paris, Denoël. BOURDIEU Pierre. 1979. La distinction. Critique sociale du jugement. Paris, Minuit.

CHADEFAUD M. 1988. Aux origines du tourisme dans les pays de l'Adour. Pau, Université de Pau et des Pays de l'Adour.

CORNU Yves. 1996. Pour une autre approche du marketing des destinations touristiques - Gérer l'offre d'une station comme on gère l'offre d'un supermarché, Les Cahiers Espaces, n 47, juin.

DUBOIS Pierre-Louis \& JOLIBERT Alain. 1998. Le marketing : fondement et pratiques. Paris, Economica.

ECHEVARRÍA Evelio. 1996. Aconcagua, Ascents and Tragedy, American Alpine Journal, p. 224.

ELIADE Mircea. 1965. Le sacré et le profane. Paris, Gallimard.

FRIEDBERG Erhard. 1993. Le pouvoir et la règle. Paris, Seuil.

GASQUES Marcus Vinicius. 2002. Montanha em fúria. Aventura e drama no cerro Aconcagua, o maior pico de América. São Paulo, Editora Globa.

GIBSON Alex. 1999. Le marketing de la destination touristique : management de la destination et gestion de la marque, Les Cahiers Espaces, n 64, décembre

HABELER Peter. 1979. Impossible Victory. Londres, Sphere Books.

HOERNER Jean-Michel. 1997. Géographie de l'industrie touristique. Paris, Ellipses.

JOUTARD Philippe. 1986. L’invention du Mont Blanc. Paris, Gallimard/Julliard.

LÉVI-STRAUSS Claude. 1962. La pensée sauvage. Paris, Plon.

\footnotetext{
${ }^{12}$ Parfois au sens physique du terme : les gelures qui feront perdre un orteil, un doigt... ou plus !
} 
MERCIER Louis-Sébastien. 1788. Tableau de Paris. Neuchãtel, 1781/Amsterdam.

NICLEVICZ Waldemar. 2007. Um sonho chamado K2. A conquista brasileira da montanha da morte. Rio de Janeiro, Editora Record, (6 $6^{\mathrm{a}}$ ed.).

PARQUE PROVINCIAL ACONCAGUA. S/D. Disponível em: http://www.aconcagua. mendoza.gov.ar/. Acesso 02 feb. 2008.

RAHARINOSY Andria \& RASPAUD Michel. 2005. Management d'une destination touristique : problématiques et perspectives opérationnelles et stratégiques de développement durable. In Les sciences de gestion au cœur du développement. Actes du Colloque international de l'INSCAE (CDROM). Antananarivo (Madagascar), 2-3-4 novembre 2005, 492 pages (16 pages).

RASPAUD Michel. 2003. Le voyage en "oxygène rare” comme expérience initiatique. L'expérimentation de la haute altitude en Himalaya, Babel. Langages - Imaginaires - Civilisations. $\mathrm{N}^{\circ}$ 8, mars 2003, p. 87-110.

SEIGNEUR Viviane. 2007. Socio-Anthropologie de la haute montagne. Paris, L’Harmattan,

WEBER S. 1998. Measuring destination attractiveness factors using a longitudinal study, in: Marketing de destination. Report of the $48^{\text {th }}$ Congress of the AIEST, 1998, Marrakech.

\section{Recebido em: 08/03/2008}

Aprovado em: 26/06/2008 